OPEN ACCESS

Edited by:

Vita Golubovskaya, ProMab Biotechnologies, United States

Reviewed by: Ravindra Deshpande, Wake Forest School of Medicine,

United States

Louise Catherine Connell, Cornell University, United States

*Correspondence: Xiaomeng $\mathrm{He}$ hxm1987@alumni.hust.edu.cn

Specialty section: This article was submitted to Gastrointestinal Cancers: Colorectal Cancer. a section of the journal

Frontiers in Oncology

Received: 12 September 2021 Accepted: 08 November 2021 Published: 25 November 2021

Citation:

Zhou H, Wang Y, Lin Y, Cai W, Li X and HeX (2021) Preliminary Efficacy and Safety of Camrelizumab in Combination With XELOX Plus Bevacizumab or Regorafenib in Patients With Metastatic Colorectal Cancer: A Retrospective Study.

Front. Oncol. 11:774445. doi: 10.3389/fonc.2021.774445

\section{Preliminary Efficacy and Safety of Camrelizumab in Combination With XELOX Plus Bevacizumab or Regorafenib in Patients With Metastatic Colorectal Cancer: A Retrospective Study}

\author{
Hong Zhou ${ }^{1}$, Yuehui Wang ${ }^{2}$, Yanfang Lin ${ }^{1}$, Wenjie Cai ${ }^{3}$, Xiaofeng $\mathrm{Li}^{4}$ and Xiaomeng $\mathrm{He}^{5 *}$ \\ 1 Department of Pharmacy, First Hospital of Quanzhou Affiliated to Fujian Medical University, Quanzhou, China, ${ }^{2}$ Department \\ of Radiology, First Hospital of Quanzhou Affiliated to Fujian Medical University, Quanzhou, China, ${ }^{3}$ Department of Radiation \\ Oncology, First Hospital of Quanzhou Affiliated to Fujian Medical University, Quanzhou, China, ${ }^{4}$ Department of Medical \\ Oncology, First Hospital of Quanzhou Affiliated to Fujian Medical University, Quanzhou, China, ${ }^{5}$ Department of Pharmacy, \\ The First Affiliated Hospital of Dalian Medical University, Dalian, China
}

Background: For a majority of patients with metastatic colorectal cancer (mCRC) with MS stable (MSS) or mismatch repair proficient (pMMR), the role of immunotherapy is undetermined. This study investigated the efficacy and safety of camrelizumab when added to XELOX chemotherapy plus bevacizumab or regorafenib as first-line therapy for mCRC.

Materials and Methods: Medical records of mCRC patients who received camrelizumab and XELOX plus bevacizumab or regorafenib at the First Hospital of Quanzhou Affiliated to Fujian Medical University between June 1, 2019, and April 30, 2021, were retrospectively collected. The objective response rate (ORR), disease control rate (DCR), progression-free survival (PFS), overall survival (OS), and side effects of the drug were recorded and reviewed.

Results: Twenty-five eligible patients received combination therapy, including bevacizumab in 19 patients and regorafenib in 6. Twenty-one patients had pMMR/MSS and one MSI-H. Of the 25 patients who could be evaluated for efficacy, $18(72 \%)$ achieved PR, 6 (24\%) achieved SD, and 1 (4\%) achieved PD. The ORR and DCR were 72\% (18/25) and 96\% (24/25), respectively. The median progression-free survival (PFS) was 11.2 months (95\% Cl 8.9-13.9), and OS had not yet been reached. The combination regimen of regorafenib in six (24\%) patients was unassociated with treatment outcomes. Most AEs were either grade 1 or 2 , and treatment-related grade 3 toxicities were observed in 8/25 (32\%) patients. 


\begin{abstract}
Conclusion: Camrelizumab combined with XELOX plus bevacizumab or regorafenib was feasible, producing high rates of responses as first-line therapy in unselected Chinese patients with MSS mCRC. The toxicities were generally tolerable and manageable. Prospective randomized trials with large sample sizes are needed to evaluate these findings.
\end{abstract}

Keywords: colorectal cancer, camrelizumab, immune checkpoint inhibitor, microsatellite stable, bevacizumab, regorafenib

\section{INTRODUCTION}

Immune checkpoint inhibitors (ICIs) have been shown to benefit patients with metastatic colorectal cancer (mCRC) with mismatch repair deficiency (dMMR) or high microsatellite instability (MSI-H) (1, 2). However, PD-1/PDL1 blockade immunotherapy is not effective in pMMR/MSS, which constitutes a large population of patients (3). Ongoing clinical trials are evaluating the efficacy of immunotherapy-based strategies, including chemotherapy, radiotherapy, MEK inhibitors, or other agents in pMMR/MSS mCRC (4). In the REGONIVO study (5), regorafenib combined with nivolumab produced an ORR of $33 \%$ (95\% CI, $15.6 \%$ to $55.3 \%$ ) in 24 patients with $\mathrm{pMMR} / \mathrm{MSS}$ refractory $\mathrm{mCRC}$, indicating that anti-angiogenic drugs may enhance the efficacy of immune checkpoint inhibitors. In addition, a single-arm phase II AVETUX trial produced a high ORR of $79.5 \%$ in 39 patients, showing the feasibility and early efficacy of avelumab and cetuximab combined with FOLFOX as first-line therapy in RAS/BRAF wildtype MCRC patients (6).

Camrelizumab (SHR-1210) a high-affinity, humanized immunoglobulin and selective IgG4-anti-PD-1 monoclonal antibody, has been approved for the treatment of classical Hodgkin's lymphoma, advanced hepatic cancer, advanced esophageal cancer, and advanced non-small-cell lung cancer in China (7). Camrelizumab is one of the most widely used antiPD-1 antibodies for various solid tumors in real-time practice owing to drug accessibility and economic pressure for patients in China. It has shown promising clinical efficacy in several kinds of solid tumors, based on positive efficacy results in clinical trials (8-11), and has also been shown to be effective in MSI-H/dMMR solid tumors (12). The combination of regorafenib and camrelizumab achieved an ORR of $25 \%$ in 16 patients with MSS refractory $\mathrm{mCRC}$, indicating the potential benefit of immunotherapy under an appropriately combined therapeutic strategy (13). Hence, we evaluated the efficacy and safety of camrelizumab when added to the first-line XELOX chemotherapy with bevacizumab or regorafenib in patients with metastatic colorectal cancer.

\section{MATERIALS AND METHODS}

\section{Patients}

The medical records of patients with mCRC who were treated with camrelizumab combined with XELOX plus bevacizumab or regorafenib at the First Hospital of Quanzhou Affiliated to Fujian
Medical University between June 1, 2019, and April 30, 2021, were retrieved. The data cutoff date was October 15, 2021. Eligibility for inclusion included histologically-confirmed metastatic colorectal cancer, treated with camrelizumab combined with XELOX plus bevacizumab or regorafenib and no prior systemic therapy, and one or more uni-dimensional measurable lesions according to Response Evaluation Criteria in Solid Tumors (RECIST) version 1.1. Prior adjuvant therapy and radiotherapy or surgery for $\mathrm{mCRC}$ were allowed. There were no exclusion criteria. This study was performed in accordance with the Declaration of Helsinki and was approved by the Ethics Review Board of the First Hospital of Quanzhou Affiliated to Fujian Medical University (Fujian Province, China).

\section{Treatment Methods}

Camrelizumab was intravenously administered at a dose of 200 mg on day 1 every 3 weeks. XELOX consisted of oxaliplatin 130 $\mathrm{mg} / \mathrm{m}^{2}$ on day 1 , followed by oral capecitabine $1,000 \mathrm{mg} / \mathrm{m}^{2}$ twice daily on days 1 through 14 (28 doses) of a 21-day cycle. Bevacizumab was administered before oxaliplatin at a dose of 7.5 $\mathrm{mg} / \mathrm{kg}$ on day 1 every 3 weeks. Regorafenib was orally administered $80 \mathrm{mg}$ once per day on day 1 through 21 in a 28day cycle. Camrelizumab was administered prior to bevacizumab and chemotherapy.

\section{Efficacy and Toxicities}

Tumor responses were evaluated after every two or three cycles of the combination therapy according to the RECIST 1.1 by computed tomography (CT) scan. The objective response rate (ORR) was calculated by pooling the complete response (CR) and partial response $(\mathrm{PR})$ rates. The disease control rate (DCR) was defined as the proportion of patients with a CR, PR, or stable disease (SD). Progression-free survival (PFS) was defined as the time from the beginning of treatment to the first documentation of disease progression, or final follow-up. Overall survival (OS) was defined as the time from the beginning of treatment to the point of death or final follow-up. Toxicities were graded according to the National Cancer Institute Common Toxicity Criteria for Adverse Events, version 5. The data cutoff date was October 15, 2021.

\section{Statistical Analysis}

Statistical analysis was performed using SPSS version 19.0(SPSS, Inc., Chicago, IL, USA). The Kaplan-Meier method was used for PFS and OS. Median follow-up times were computed by the reverse Kaplan-Meier method. Log-Rank test was performed to compare the different groups for PFS univariate analysis. A twotailed $\mathrm{p}<0.05$ was considered statistically significant in all tests. 


\section{RESULTS}

\section{Patient Characteristics}

The baseline characteristics of the 25 patients with mCRC are shown in Table 1. The median age was 64 years (range 43-86 years). Among all patients, 13 (52\%) were male and 12 (48\%) female. Fifteen (60\%) were ECOG PS 0 and 8 (32\%) ECOG PS 1. In total, $18(72 \%)$ patients were left-sided primary colorectal cancer, and seven (28\%) patients right-sided primary colon cancer. Fourteen (56\%) patients had Liver metastases. Twentyone $(84 \%)$ patients were confirmed as pMMR/MSS. Among the 25 patients, 19 (76\%) patients received combination immunotherapy of bevacizumab, and 6 (24\%) of regorafenib (Table 2).

\section{Efficacy}

Of the 25 patients, none achieved CR; 18(72\%) experienced partial responses and 6(24\%) experienced stable disease as best responses, while one (4\%) patient had progressive disease. The ORR and DCR were 72\% (18/25) and 96\% (24/25), respectively (Table 3). The median follow-up for overall survival was 11.5months (95\% CI10.3-12.7). The median progression-free survival(mPFS) was 11.2 months (95\% CI 8.9-13.9) (Figure 1A). The OS was still immature and one-year OS rates were $70.4 \%$ (95\% CI 43.7-86.1) (Figure 1B). In addition, the mPFS of the regorafenib-containing regimen was 9.6 months and PFS of

TABLE 1 | Baseline Characteristics.

\begin{tabular}{lc}
\hline Characteristics & Patients N (\%) \\
\hline Age (year) & \\
Median age (range) & $64(43-86)$ \\
$\geq 60$ & $15(60)$ \\
$<60$ & $10(40)$ \\
Sex & \\
Male & $13(52)$ \\
Female & $12(48)$ \\
ECOG PS & \\
0 & $15(60)$ \\
1 & $8(32)$ \\
2 & $2(8)$ \\
Primary tumor location & \\
Colon & $14(56)$ \\
Right-side & $7(28)$ \\
Left-side & $7(28)$ \\
Rectum & $11(44)$ \\
Type of metastasis & \\
With liver metastasis & $14(56)$ \\
Without liver metastasis & $11(44)$ \\
Site of distant metastasis & \\
Liver & $14(56)$ \\
Lung & $7(28)$ \\
Lymph nodes & $6(24)$ \\
Peritoneum & $6(24)$ \\
Peritoneal cavity & $8(32)$ \\
Other & $5(20)$ \\
MMR or MSI status & \\
pMMR or MSS & $21(84)$ \\
dMMR or MSI-H & $1(4)$ \\
Unknown & $3(12)$ \\
\hline
\end{tabular}

ECOG PS, Eastern Cooperative Oncology Group performance score. bevacizumab-containing regimen has not yet been reached, although the difference was considered not statistically significant $(\mathrm{p}=0.08$, Figure $\mathbf{1 C})$. The difference in patients with liver metastasis did not reach statistical significance compared with patients without liver metastasis (11.2 vs. 10.9 months, $\mathrm{p}=0.81$, Figure 1D).

\section{Safety}

All 25 patients were assessed for toxicities. The overall incidence of any grade toxicity was $72 \%(18 / 25)$. Common treatmentrelated adverse events (AEs) of any grade were neutropenia (36\%) reactive cutaneous capillary endothelial proliferation $(32 \%)$, decreased platelet count $(28 \%)$, hand-foot syndrome (28\%), and liver dysfunction (16\%). Grade $\geq 3$ treatmentrelated AEs occurred in $8(32 \%)$ patients. They included neutropenia, gastric hemorrhage, hand-foot syndrome, hyperglycemia, and elevated ALT. A fatal event of gastrointestinal perforation complicated by febrile neutropenia occurred in a patient treated with regorafenib. Treatment was switched from capecitabine to raltitrexed due to intolerable grade 3 hand-foot syndrome in three $(12 \%)$ patients. The most common camrelizumab-related adverse events were reactive cutaneous capillary endothelial proliferation (32\%) and thyroid dysfunction (24.0\%), and all of them were either grade 1 or 2 . A new camrelizumab-related adverse event, mild bilateral optic disc disease, was observed. Details of the adverse events are presented in Table 4.

\section{DISCUSSION}

Although recently, rapid development has been made in the field of immunotherapy $(14,15)$, the first-line standard treatment for metastatic colorectal cancer (mCRC) is $5 \mathrm{FU}$-based chemotherapies, with or without anti-angiogenic agents. ICI monotherapy is efficacious in the treatment of mCRC with dMMR/MSI-H. The role of the combination of PD-1 blockade with VEGF inhibition has been investigated in MSS mCRC. Adding atezolizumab to $\mathrm{FP} / \mathrm{BEV}$ (standard of care) as first-line maintenance treatment for patients with BRAF wild-type mCRC did not lead to improvement in the outcomes for efficacy (16). However, in the AVETUX trial, avelumab and cetuximab in combination with FOLFOX in patients with previously untreated mCRC produced a high response rate of $79.5 \%$, disease control rate of $92.3 \%$ and mPFS of 11.1 months in 39 patients with RAS/ BRAF wild-type MSS mCRC (6). In this study, a high ORR of $72 \%$ and a DCR of $96 \%$ were recorded. The mPFS of 11.2 months is as long as that of the AVETUX trial (11.1 months), which is much better than that of bevacizumab plus XELOX chemotherapy (9.4 months) as first-line therapy in patients with mCRC (17). The main factors leading to different efficacies may be that populations in the AVETUX trial included more patients with left-sided tumors (91\%) compared to our study (68.8\%), and all patients were RAS/BRAF wild-type. Patients with RAS with left-sided mCRC had significantly superior PFS, OS, and ORR compared with patients with 
TABLE 2 | Characteristics of individual patients.

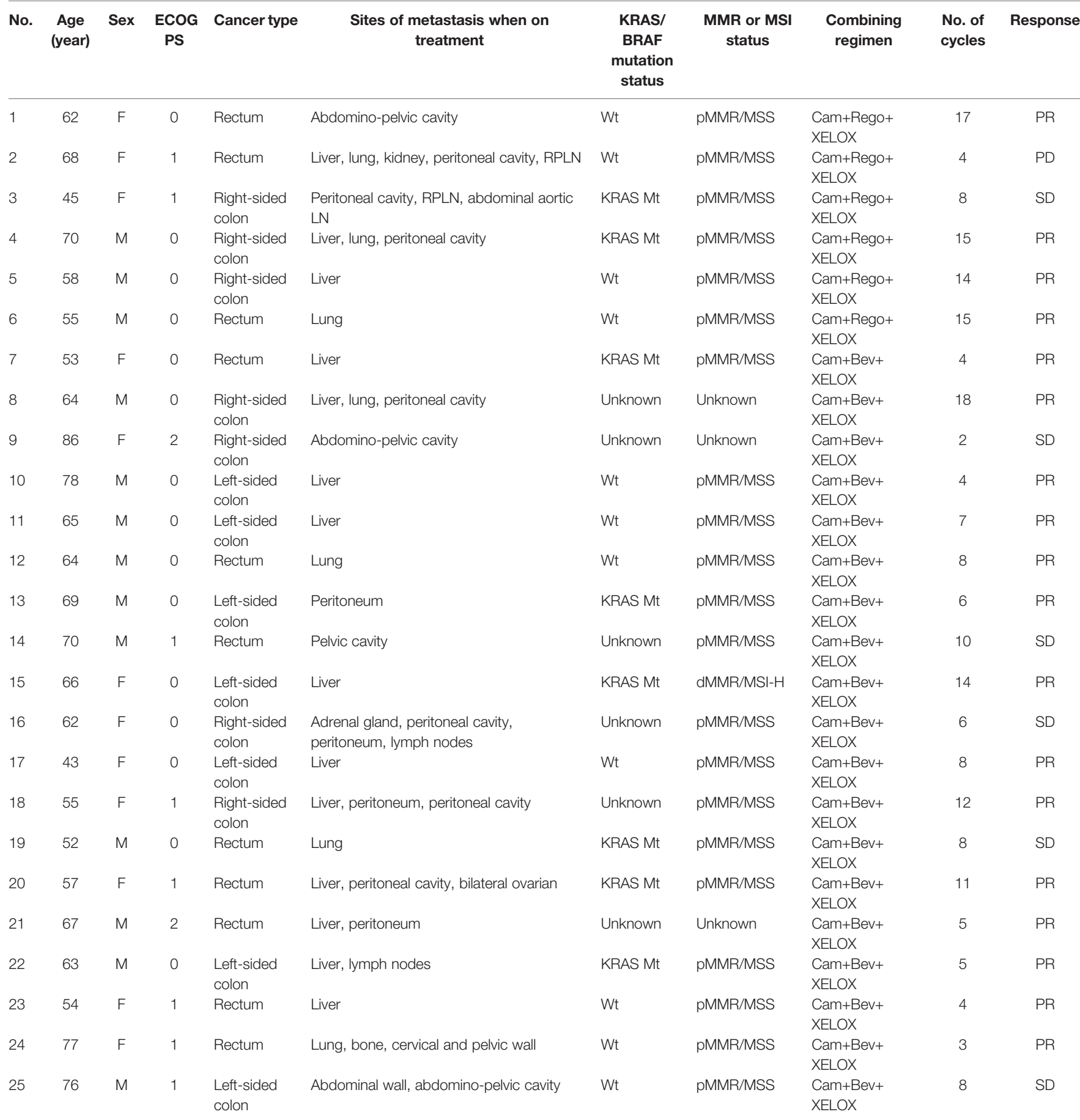

ECOG PS, Eastern Cooperative Oncology Group performance status; F, female; M, male; Mt, mutant; Wt, wild-type; Cam, camrelizumab; Bev, bevacizumab; Rego, regorafenib; XELOX, capecitabine and oxaliplatin; PR, partial response; $S D$, stable disease; $P D$, progression of disease; RPLN, retroperitoneal lymph node.

right-sided tumors (18), and cetuximab plus FOLFIRI versus bevacizumab as first-line treatment clearly benefitted patients with left-sided tumors $(18,19)$.

Several studies have suggested that liver metastases are predictive of a lack of benefit from PD-1/PD-L1 inhibitors in MSS mCRC $(5,20)$. The liver is considered an immunologicallytolerant organ that is characterized by a much higher proportion of immunosuppressive cells (21). In the present study, the difference in patients with liver metastasis was considered not statistically significant compared with those without liver metastasis, probably due to the small sample size.

Recently, in the REGONIVO trial, the combination of regorafenib and nivolumab achieved a robust response rate of $33 \%$ in pMMR/MSS refractory mCRC (5). More and more 
TABLE 3 | Tumor response.

\begin{tabular}{lc}
\hline Response & $\begin{array}{c}\text { Patients } \mathbf{~} \\
\text { (\%) }\end{array}$ \\
\hline CR & 0 \\
PR & $18(72)$ \\
SD & $6(24)$ \\
PD & $1(4)$ \\
ORR & $18(72)$ \\
DCR & $24(96)$ \\
\hline
\end{tabular}

$C R$, complete response; $P R$, partial response; $S D$-stable disease; $P D$, progression of disease; ORR, objective response rate; $D C R$, disease control rate.

research focused on the regorafenib plus ICIs in MSS refractory $\mathrm{mCRC}$, the current conclusion is controversial due to the small sample size and inconsistency $(5,13,22-24)$. In this study, we observed that the mPFS of regorafenib-containing therapies was not as long as that of bevacizumab-containing therapies. Moreover, capecitabine-related hand-foot syndrome (HFS), one of the causes of the switch in three treatments, was in a regorafenib-containing regimen. HFS is a common skin reaction to capecitabine with rates of any grade, of $22 \%-77 \%$ (25). Similarly, regorafenib-associated hand-foot skin reactions occurred at a rate of $61 \%$ overall and $20 \%$ at grade $3(26,27)$. Regorafenib combined with capecitabine treatment should be used cautiously due to the risk of overlapping skin toxicity. Therefore, this might suggest that as first-line therapy for mCRC, regorafenib may not be a suitable choice for combination with XELOX chemotherapy in future trials.
TABLE 4 | Adverse events.

\begin{tabular}{lccc}
\hline Adverse event & $\begin{array}{c}\text { Grade 1-2, N } \\
(\mathbf{\%})\end{array}$ & $\begin{array}{c}\text { Grade } \geq \mathbf{3}, \mathbf{N} \\
\mathbf{( \% )}\end{array}$ & $\begin{array}{c}\text { Any grade, N } \\
\mathbf{( \% )}\end{array}$ \\
\hline Neutropenia & $8(32)$ & $1(4)$ & $9(36)$ \\
Decreased platelet & $6(24)$ & $1(4)$ & $7(28)$ \\
count & & & \\
Nausea and Vomiting & $1(4)$ & $1(4)$ & $2(8)$ \\
Liver dysfunction & $2(8)$ & $2(8)$ & $4(16)$ \\
Hand-foot syndrome & $4(16)$ & $3(12)$ & $7(28)$ \\
Gastric hemorrhage & 0 & $1(4)$ & $1(4)$ \\
Diarrhea & $1(4)$ & $1(4)$ & $2(8)$ \\
Fever & $2(8)$ & $1(4)$ & $3(12)$ \\
Hyperthyroidism & $4(16)$ & 0 & $4(16)$ \\
Hypothyroidism & $2(8)$ & 0 & $2(8)$ \\
Hyperglycemia & $1(4)$ & $1(4)$ & $2(8)$ \\
RCCEP & $8(32)$ & 0 & $8(32)$ \\
Vision changes & $1(4)$ & 0 & $1(4)$ \\
Myocarditis & $2(8)$ & 0 & $2(8)$ \\
Infusion-related & $2(8)$ & 0 & $2(8)$ \\
reactions & $16(64)$ & $8(32)$ & $18(72)$ \\
ALL & & & \\
\hline RCCEP, reative & & 0 &
\end{tabular}

RCCEP, reactive cutaneous capillary endothelial proliferation.

The combination strategy indicated a potential benefit in terms of ORR and PFS with a acceptable safety profile. Despite its strengths, there are some limitations to consider. First, this is a retrospective pilot study with a small sample size, reflecting its preliminary nature. Second, although antitumor response was observed in mCRC in spite of RAS mutations, a small number of patients had unknown RAS or BRAF status before the beginning

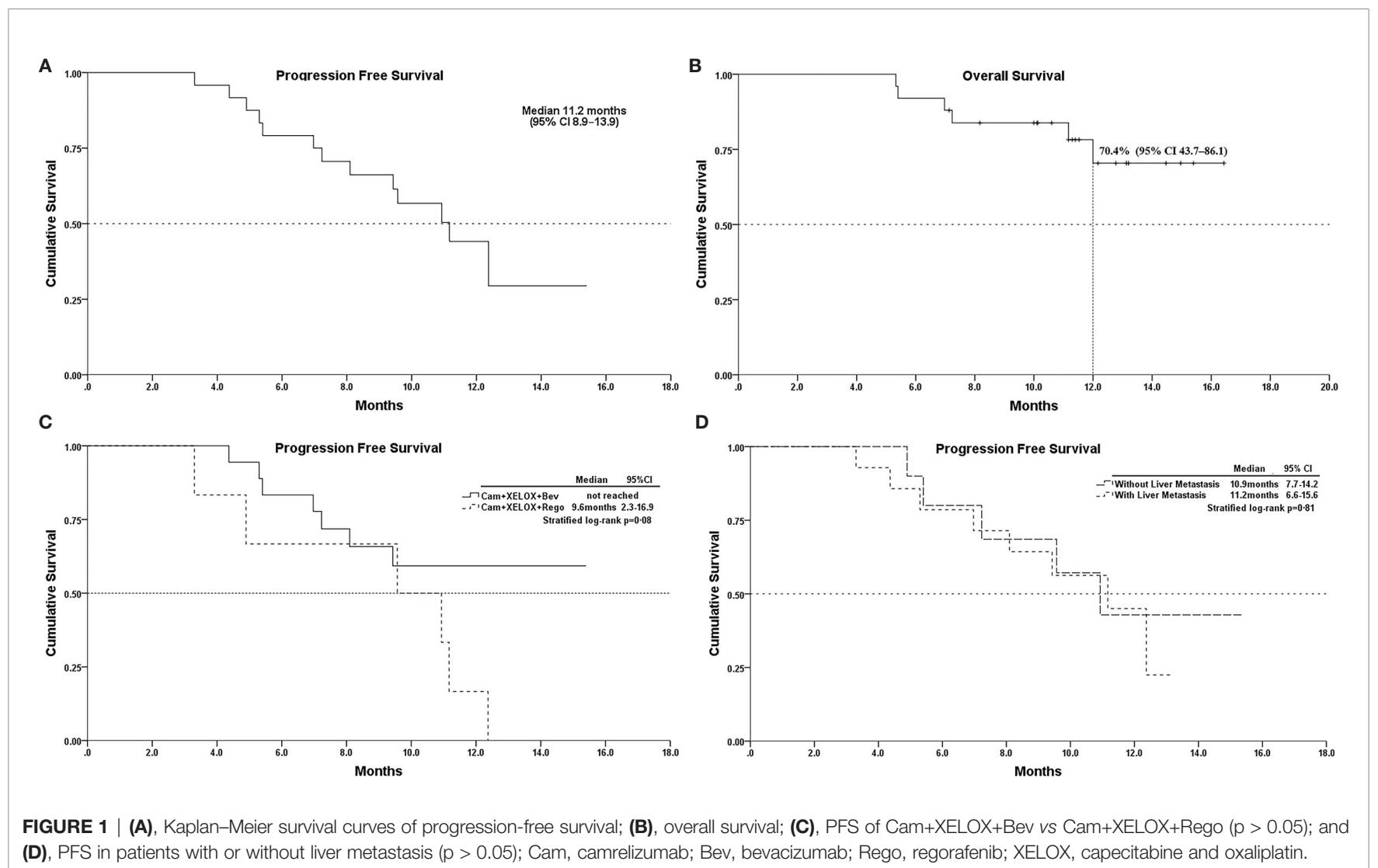


of the combination treatment. Besides, the MMR or MSI status of three patients was unknown. Finally, even if the same chemotherapy regimen was adopted, different courses of treatment and varying follow-up intervals may have increased the heterogeneity. Thus, the findings need to be further assessed in a large prospective study.

In conclusion, our study differs from previous immunotherapy study in MSS mCRC based on the study populations and the novel combination regimen. In present study showed the addition of camrelizumab to the first-line XELOX chemotherapy with bevacizumab have demonstrated high response rates, and this immunotherapy combination was practical and helpful in unselected patients with mCRC. Further randomized trials with large sample sizes for this combination strategy are warranted.

\section{DATA AVAILABILITY STATEMENT}

The raw data supporting the conclusions of this article will be made available by the authors, without undue reservation.

\section{ETHICS STATEMENT}

This study was approved by the Ethics Review Board of the First Hospital of Quanzhou Affiliated to Fujian Medical University,

\section{REFERENCES}

1. Le DT, Uram JN, Wang H, Bartlett BR, Kemberling H, Eyring AD, et al. PD-1 Blockade in Tumors With Mismatch-Repair Deficiency. N Engl J Med (2015) 372(26):2509-20. doi: 10.1056/NEJMoa1500596

2. Overman MJ, McDermott R, Leach JL, Lonardi S, Lenz HJ, Morse MA, et al. Nivolumab in Patients With Metastatic DNA Mismatch Repair-Deficient or Microsatellite Instability-High Colorectal Cancer (Checkmate 142): An Open-Label, Multicentre, Phase 2 Study. Lancet Oncol (2017) 18(9):118291. doi: 10.1016/S1470-2045(17)30422-9

3. Overman MJ, Lonardi S, Wong KYM, Lenz HJ, Gelsomino F, Aglietta M, et al. Durable Clinical Benefit With Nivolumab Plus Ipilimumab in DNA Mismatch Repair-Deficient/Microsatellite Instability-High Metastatic Colorectal Cancer. J Clin Oncol (2018) 36(8):773-9. doi: 10.1200/ JCO.2017.76.9901

4. Wei XL, Zhang Y, Zhao HY, Wang ZQ, Li YH, Wang F, et al. A Phase I Study of SHR7390 Plus Camrelizumab in Advanced/Metastatic Colorectal Cancer. J Clin Oncol (2021) 39(15_suppl):e15553. doi: 10.1200/JCO.2021.39. 15_suppl.e15553

5. Fukuoka S, Hara H, Takahashi N, Kojima T, Kawazoe A, Asayama M, et al. Regorafenib Plus Nivolumab in Patients With Advanced Gastric or Colorectal Cancer: An Open-Label, Dose-Escalation, and Dose-Expansion Phase Ib Trial (Regonivo, Epoc1603). J Clin Oncol (2020) 38(18):2053-61. doi: 10.1200/ JCO.19.03296

6. Stein A, Binder M, Goekkurt E, Lorenzen S, Riera-Knorrenschild J, Depenbusch R, et al. Avelumab and Cetuximab in Combination With Folfox in Patients With Previously Untreated Metastatic Colorectal Cancer (mCRC): Final Results of the Phase Ii Avetux Trial (Aio-Krk-0216). J Clin Oncol (2020) 38(4_suppl):96. doi: 10.1200/JCO.2020.38.4_suppl.96

7. Song H, Liu X, Jiang L, Li F, Zhang R, Wang P. Current Status and Prospects of Camrelizumab, a Humanized Antibody Against Programmed Cell Death Receptor 1. Recent Pat Anticancer Drug Discov (2021) 16(3):312-32. doi: $10.2174 / 1574892816666210208231744$
East Road 250, Quanzhou 362000, Fujian Province, China. Written informed consent for participation was not required for this study, in accordance with the national legislation and institutional requirements.

\section{AUTHOR CONTRIBUTIONS}

WC, XL, and XH designed the project. HZ, YL, and XH collected patients' information and wrote the manuscript. WC and XL provided study material or patient. YW evaluated tumor responses. All authors contributed to the article and approved the submitted version.

\section{ACKNOWLEDGMENTS}

We sincerely thank Hongyue Lin, Zhiqiang Lin, Zhihang Lin, Lili Cai, Lei Hong, and Zede Ye who offered their assistance in preparing the manuscript.

\section{SUPPLEMENTARY MATERIAL}

The Supplementary Material for this article can be found online at: https://www.frontiersin.org/articles/10.3389/fonc.2021.774445/ full\#supplementary-material

8. Liu J, Liu Q, Li Y, Li Q, Su F, Yao H, et al. Efficacy and Safety of Camrelizumab Combined With Apatinib in Advanced Triple-Negative Breast Cancer: An Open-Label Phase Ii Trial. J Immunother Cancer (2020) 8(1):e000696. doi: 10.1136/jitc-2020-000696

9. Lan C, Huang X, Shen JX, Wang Y, Xiong Y, Li J, et al. Camrelizumab Plus Apatinib in Patients With Advanced Cervical Cancer: A Multicenter, OpenLabel, Single-Arm, Phase Ii Trial. J Clin Oncol (2020) 38(15_suppl):6021. doi: 10.1200/JCO.2020.38.15_suppl.6021

10. Fan Y, Zhao J, Wang Q, Huang D, Li X, Chen J, et al. Camrelizumab Plus Apatinib in Extensive-Stage SCLC (PASSION): A Multicenter, Two-Stage, Phase 2 Trial. J Thorac Oncol (2021) 16(2):299-309. doi: 10.1016/ j.jtho.2020.10.002

11. Chen X, Wu X, Wu H, Gu Y, Shao Y, Shao Q, et al. Camrelizumab Plus Gemcitabine and Oxaliplatin (Gemox) in Patients With Advanced Biliary Tract Cancer: A Single-Arm, Open-Label, Phase Ii Trial. J Immunother Cancer (2020) 8(2):e001240. doi: 10.1136/jitc-2020-001240

12. Chen J, Quan M, Chen Z, Zeng T, Li Y, Zhou Y, et al. Camrelizumab in Advanced or Metastatic Solid Tumour Patients With DNA Mismatch Repair Deficient or Microsatellite Instability High: An Open-Label Prospective Pivotal Trial. J Cancer Res Clin Oncol (2020) 146(10):2651-7. doi: 10.1007/ s00432-020-03251-5

13. Jiang FE, Zhang HJ, Yu CY, Liu AN. Efficacy and Safety of Regorafenib or Fruquintinib Plus Camrelizumab in Patients With Microsatellite Stable and/ or Proficient Mismatch Repair Metastatic Colorectal Cancer: An Observational Pilot Study. Neoplasma (2021) 68(4):861-6. doi: 10.4149/ neo_2021_201228N1415

14. Benson AB, Venook AP, Al-Hawary MM, Arain MA, Chen YJ, Ciombor KK, et al. Colon Cancer, Version 2.2021, NCCN Clinical Practice Guidelines in Oncology. J Natl Compr Canc Netw (2021) 19(3):329-59. doi: 10.6004/jnccn.2021.0012

15. Damilakis E, Mavroudis D, Sfakianaki M, Souglakos J. Immunotherapy in Metastatic Colorectal Cancer: Could the Latest Developments Hold the Key to Improving Patient Survival? Cancers (Basel) (2020) 12(4):889. doi: 10.3390/ cancers12040889 
16. Grothey A, Tabernero J, Arnold D, De Gramont A, Ducreux MP, O’Dwyer PJ, et al. Fluoropyrimidine (FP) + Bevacizumab (BEV) + Atezolizumab vs FP/ $\mathrm{BEV}$ in BRAFwt Metastatic Colorectal Cancer (mCRC): Findings From Cohort 2 of MODUL - A Multicentre, Randomized Trial of BiomarkerDriven Maintenance Treatment Following First-Line Induction Therapy. Ann Oncol (2018) 29:viii714-5. doi: 10.1093/annonc/mdy424.020

17. Saltz LB, Clarke S, Díaz-Rubio E, Scheithauer W, Figer A, Wong R, et al. Bevacizumab in Combination With Oxaliplatin-Based Chemotherapy as First-Line Therapy in Metastatic Colorectal Cancer: A Randomized Phase Iii Study. J Clin Oncol (2008) 26(12):2013-9. doi: 10.1200/ JCO.2007.14.9930

18. Tejpar S, Stintzing S, Ciardiello F, Tabernero J, Van Cutsem E, Beier F, et al. Prognostic and Predictive Relevance of Primary Tumor Location in Patients With Ras Wild-Type Metastatic Colorectal Cancer: Retrospective Analyses of the Crystal and Fire-3 Trials. JAMA Oncol (2017) 3(2):194-201. doi: 10.1001/ jamaoncol.2016.3797

19. Heinemann V, Von Weikersthal LF, Decker T, Kiani A, Vehling-Kaiser U, AlBatran SE, et al. Folfiri Plus Cetuximab Versus Folfiri Plus Bevacizumab as First-Line Treatment for Patients With Metastatic Colorectal Cancer (Fire-3): A Randomised, Open-Label, Phase 3 Trial. Lancet Oncol (2014) 15(10):106575. doi: 10.1016/S1470-2045(14)70330-4

20. Fakih M, Sandhu J, Wang C, Ouyang C. Response to PD-1 and PD-L1 Based Immunotherapy in MSS Advanced Colorectal Cancer is Impacted by Metastatic Disease Sites. J Clin Oncol (2021) 39(3_suppl):72. doi: 10.1200/ JCO.2021.39.3_suppl.72

21. Kubes P, Jenne C. Immune Responses in the Liver. Annu Rev Immunol (2018) 36:247-77. doi: 10.1146/annurev-immunol-051116-052415

22. Wang C, Chevalier D, Saluja J, Sandhu J, Lau C, Fakih M. Regorafenib and Nivolumab or Pembrolizumab Combination and Circulating Tumor DNA Response Assessment in Refractory Microsatellite Stable Colorectal Cancer. Oncologist (2020) 25(8):e1188-94. doi: 10.1634/theoncologist.2020-0161

23. Li J, Cong L, Liu J, Peng L, Wang J, Feng A, et al. The Efficacy and Safety of Regorafenib in Combination With Anti-Pd-1 Antibody in Refractory
Microsatellite Stable Metastatic Colorectal Cancer: A Retrospective Study. Front Oncol (2020) 10:594125. doi: 10.3389/fonc.2020.594125

24. Wang F, He MM, Yao YC, Zhao X, Wang ZQ, Jin Y, et al. Regorafenib Plus Toripalimab in Patients With Metastatic Colorectal Cancer: A Phase Ib/II Clinical Trial and Gut Microbiome Analysis. Cell Rep Med (2021) 2 (9):100383. doi: 10.1016/j.xcrm.2021.100383

25. Kwakman JJM, Elshot YS, Punt CJA, Koopman M. Management of Cytotoxic Chemotherapy-Induced Hand-Foot Syndrome. Oncol Rev (2020) 14(1):4422. doi: 10.4081/oncol.2020.442

26. Belum VR, Wu S, Lacouture ME. Risk of Hand-Foot Skin Reaction With the Novel Multikinase Inhibitor Regorafenib: A Meta-Analysis. Inves New Drugs (2013) 31(4):1078-86. doi: 10.1007/s10637-013-9977-0

27. McLellan B, Ciardiello F, Lacouture ME, Segaert S, Van Cutsem E. Regorafenib-Associated Hand-Foot Skin Reaction: Practical Advice on Diagnosis, Prevention, and Management. Ann Oncol (2015) 26(10):201726. doi: 10.1093/annonc/mdv244

Conflict of Interest: The authors declare that the research was conducted in the absence of any commercial or financial relationships that could be construed as a potential conflict of interest.

Publisher's Note: All claims expressed in this article are solely those of the authors and do not necessarily represent those of their affiliated organizations, or those of the publisher, the editors and the reviewers. Any product that may be evaluated in this article, or claim that may be made by its manufacturer, is not guaranteed or endorsed by the publisher.

Copyright (C) 2021 Zhou, Wang, Lin, Cai, Li and He. This is an open-access article distributed under the terms of the Creative Commons Attribution License (CC BY). The use, distribution or reproduction in other forums is permitted, provided the original author(s) and the copyright owner(s) are credited and that the original publication in this journal is cited, in accordance with accepted academic practice. No use, distribution or reproduction is permitted which does not comply with these terms. 